\title{
Correction to: Metachromatic leukodystrophy with late adult-onset: diagnostic clues and differences from other genetic leukoencephalopathies with dementia
}

\author{
Chiara Benzoni $^{1}$ (D) Marco Moscatelli $^{2} \cdot$ Silvia Fenu $^{1} \cdot$ Anna Venerando $^{3} \cdot$ Ettore Salsano $^{1,4}$
}

Published online: 23 February 2021

๑) Springer-Verlag GmbH Germany, part of Springer Nature 2021

\section{Correction to: Journal of Neurology \\ https://doi.org/10.1007/s00415-020-10374-9}

The original version of this article unfortunately contained a mistake. The subtitle of the lower part of the table 1 is "Genetic leukoencephalopathies with dementia as predominant features" and not "Genetic leukoencephalopathies with dementia as prominent features".

The correct version of Table 1 is given in the next page.

The original article has been corrected.

The original article can be found online at https://doi.org/10.1007/ s00415-020-10374-9.

Chiara Benzoni

chiara.benzoni1@unimi.it

1 Unit of Rare Neurodegenerative and Neurometabolic Diseases, Fondazione IRCCS Istituto Neurologico Carlo Besta, Via Celoria 11, Milan 20133, Italy

2 Unit of Neuroradiology, Fondazione IRCCS Istituto Neurologico Carlo Besta, Milan, Italy

3 Unit of Medical Genetics and Neurogenetics, Fondazione IRCCS Istituto Neurologico Carlo Besta, Milan, Italy

4 Neuroscience PhD Program, University of Milano-Bicocca, Monza, Italy 


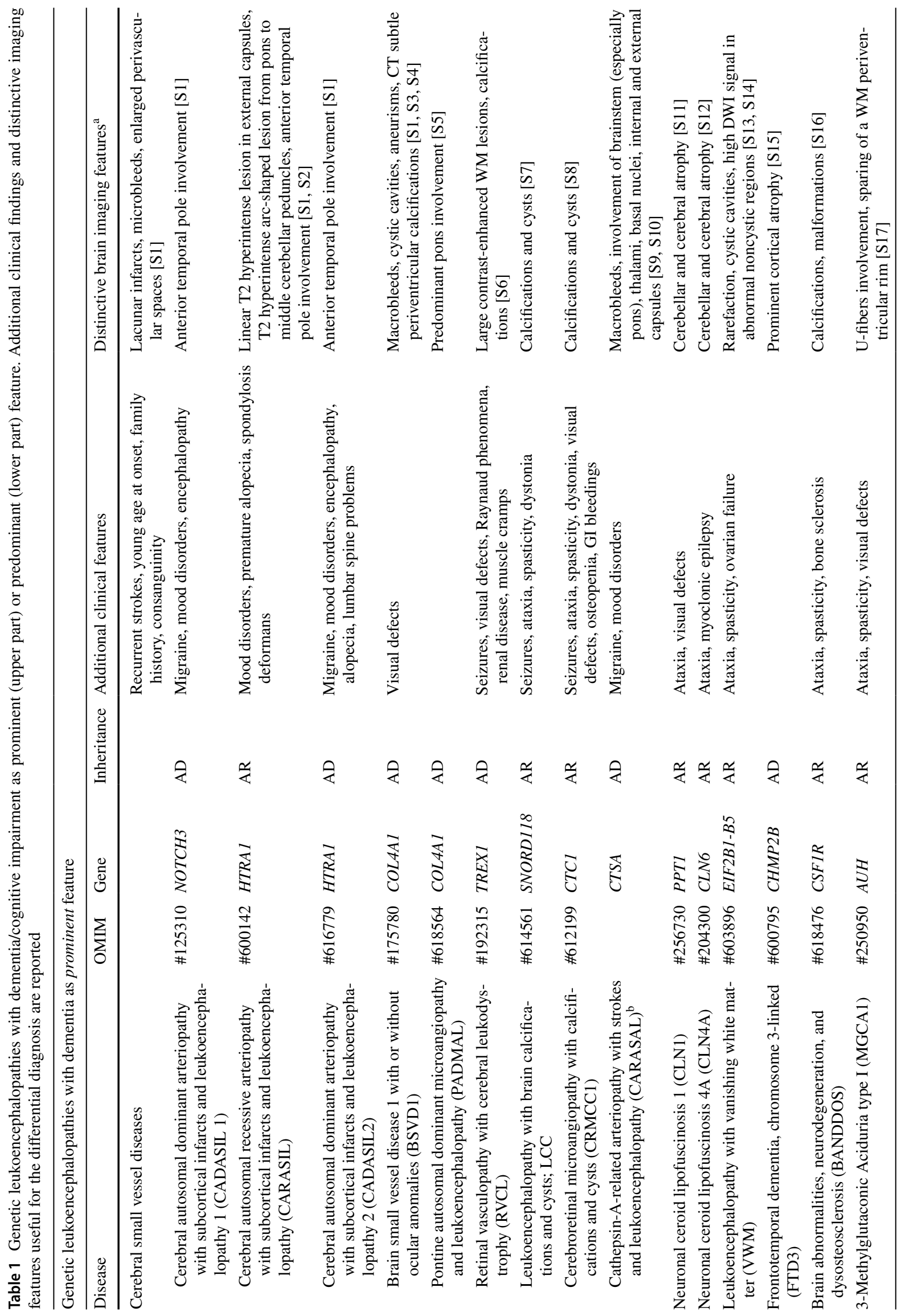




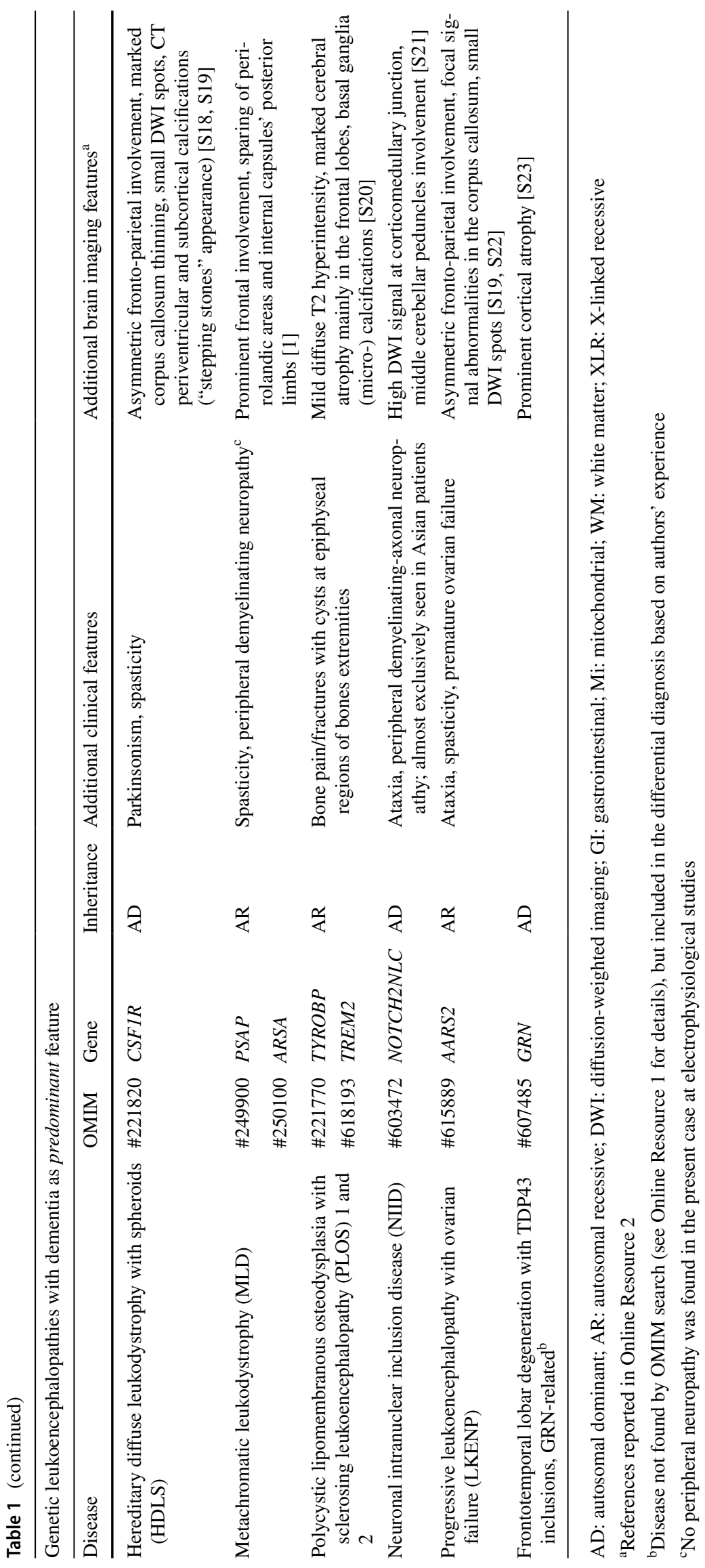

